\title{
Análise da efetividade de componente curricular para motivar colaboradores em projetos de Software Público
}

\author{
João C. Sedraz Silva ${ }^{1}$, Alex Sandro Gomes ${ }^{2}$, Jorge L. C. Ramos ${ }^{1}$, \\ Fernando d. F. de Souza ${ }^{2}$, Rodrigo Lins Rodrigues ${ }^{3}$ \\ ${ }^{1}$ Universidade Federal do Vale do São Francisco \\ Juazeiro - BA - Brasil \\ ${ }^{2}$ Universidade Federal de Pernambuco \\ Recife-PE, Brasil \\ ${ }^{3}$ Universidade Federal Rural de Pernambuco \\ Recife-PE, Brasil. \\ \{joao.sedraz,jorge.cavalcanti\}@univasf.edu.br, \\ $\{$ asg, fdfd\}@cin.ufpe.br, rodrigo.linsrodrigues@ufrpe.br
}

\begin{abstract}
The aim of this study was to analyze the effectiveness of the Núcleo Temático do Software Público Brasileiro (Núcleo SPB) to motivate students to collaborate in the projects of Portal do Software Público Brasileiro. The Núcleo SPB was the first course offered by a public university in Brazil exclusively for the promotion of Software Público Brasileiro. The results indicate that the discipline promoted significant gains in student motivation, contributing to learning and reflection of the benefits associated with the Software Público Brasileiro.
\end{abstract}

Resumo. O objetivo deste estudo foi analisar a efetividade do Núcleo Temático do Software Público Brasileiro (Núcleo SPB) para motivar estudantes a colaborarem em projetos do Portal do Software Público Brasileiro. O Núcleo SPB foi a primeira disciplina ofertada por uma universidade pública no Brasil, exclusivamente, para a promoção do Software Público Brasileiro. Os resultados indicam que a disciplina promoveu ganhos significativos na motivação dos estudantes, colaborando para o aprendizado e a reflexão dos benefícios associados ao Software Público Brasileiro.

\section{Introdução}

A adoção de Software Livre por instituições públicas oferece várias vantagens se comparada aos modelos fundamentados em software proprietário. Entre os benefícios técnicos das soluções livres estão a flexibilidade para adequar às necessidades específicas, a facilidade de auditar o código-fonte e a redução do risco de bloqueio de fornecedores. Em relação às vantagens econômicas, favorecem o desenvolvimento da indústria local e eliminam as despesas com licenças. E, ainda, como benefício social, essa solução de software constrói um patrimônio comum de toda sociedade na forma de conhecimento. Com isso, a tecnologia torna-se acessível a todos e não apenas a um grupo restrito [González et al. 2007]. 
Cientes dos benefícios técnicos, econômicos e sociais, nos últimos anos, diversos governos têm institucionalizado a adoção de Software Livre. Destaque no cenário nacional, o Portal do Software Público Brasileiro - Portal SPB (www.softwarepublico.gov.br) representa uma das maiores iniciativas no mundo de reconhecimento do valor desse tipo de solução para a sociedade. O portal é o ambiente público oficial para a liberação, o compartilhamento e o desenvolvimento de soluções livres certificadas como Software Público Brasileiro (SPB) [Terceiro et al. 2015].

Os softwares do Portal SPB oferecem soluções economicamente viáveis para milhares de municípios brasileiros que, por limitação de recursos, dificilmente teriam condições de adquirir e sustentar custos com licenças proprietárias. As novas exigências legais associadas à transparência pública reforçam ainda mais a importância do SPB e, simultaneamente, criam diversas oportunidades para empresas nacionais no desenvolvimento e prestação de serviços, colaborando para geração de emprego e distribuição de renda [Silva 2014].

Embora exista uma crescente demanda, ainda, é difícil atrair colaboradores em projetos de SPB, principalmente, em razão do limitado número de profissionais com experiência e conhecimento suficientes para reconhecerem as vantagens e atuarem no desenvolvimento ou prestação de serviço desse tipo de software [Silva 2014].

Uma alternativa para esse problema é a integração com as Instituições de Ensino Superior (IES) que, em todo o mundo, constituem a principal fonte de desenvolvedores do Software Livre [González et al. 2007]. No Brasil, os estudantes de graduação representam a maior parcela de colaboradores ativos em comunidades de Software Livre [Pinto e Kamel 2014], mas o potencial dos acadêmicos pouco tem sido explorado para a sustentabilidade e ampliação das soluções disponibilizadas no Portal SPB.

Como uma proposta para motivar universitários a colaborarem em projetos de SPB e reduzir a carência de profissionais com competência nesse tipo software, em 2014, a Universidade Federal do Vale do São Francisco (Univasf) ofertou a primeira disciplina do Brasil planejada, exclusivamente, para a promoção do SPB. A componente curricular chama-se Núcleo Temático do Software Público Brasileiro (Núcleo SPB).

O Núcleo SPB é fruto de um Acordo de Cooperação Técnica entre a Univasf e o Ministério do Planejamento, órgão responsável pela gestão do Portal SPB. Após o projeto piloto desenvolvido na Univasf, por meio de chamada pública [Brasil 2015], o governo federal selecionou outras IES que poderão adotar o modelo do Núcleo SPB como estratégia para promoção do SPB no âmbito de seus cursos de graduação. Mas, antes da replicação do modelo, é necessária a análise da seguinte questão: Seria o Núcleo SPB uma abordagem efetiva para motivar estudantes universitários a colaborarem em comunidades de Software Público Brasileiro?

Diante disso, o objetivo deste trabalho foi analisar a efetividade do Núcleo SPB, compreendida como a capacidade da disciplina em produzir um incremento na motivação dos estudantes para colaborarem em projetos do Portal SPB.

Além desta introdução, o trabalho está organizado com mais quatro seções, que apresentam fatores que motivam os colaboradores de Software Livre, o método utilizado, a discussão dos resultados e as considerações finais da pesquisa. 
V Congresso Brasileiro de Informática na Educação (CBIE 2016)

Anais do XXII Workshop de Informática na Escola (WIE 2016)

\section{Fatores que motivam os colaboradores de Software Livre}

O Software Livre se constitui como um conjunto de componentes interrelacionados que funcionam para atender a determinado objetivo e, regido por regras próprias de sua comunidade, o seu desenvolvimento é fundamentado por processos centrados na colaboração. Essas características fazem com que o Software Livre seja classificado como um sistema sociotécnico, onde o desenvolvimento não é determinístico e depende do comportamento dos operadores humanos.

O sucesso de um sistema sociotécnico está intimamente associado aos fatores motivacionais dos seus desenvolvedores. A previsão dos efeitos desses fatores nos sistemas é muito difícil de ser estabelecida. Segundo Sommerville (2011), para auxiliar o entendimento de sistemas dessa natureza, várias metodologias foram desenvolvidas, com destaque para a Soft Systems Metodology.

A partir dos elementos da metodologia Soft Systems Metodology, Feller e Fitzgerald (2000) apresentaram uma classificação das motivações dos colaboradores de Software Livre, que considera três grandes áreas - tecnológica, econômica e sociopsicológica.

\subsection{Motivações tecnológicas}

A busca por novas habilidades e competências é uma importante motivação para participação em comunidades de Software Livre. Os projetos oferecem inúmeras oportunidades para aprendizagem, em que programadores estudam profundamente $\mathrm{o}$ código, implementam novas funcionalidades e partilham o conhecimento. Esse aspecto é crucial para a indústria de software, que é impulsionada por inovações incrementais e sequenciais. Esse processo tende a produzir software de alta qualidade e tecnologia de ponta [Bonaccorsi e Rossi 2006].

Scacchi et al. (2006) destacam que, no desenvolvimento de Software Livre, diferente de projetos convencionais, o desenvolvedor tem liberdade para desempenhar funções que deseja, não sendo obrigado a realizar tarefa que o desagrada. Dessa forma, o desenvolvedor está mais propenso a se dedicar ao trabalho e ser reconhecido como colaborador de confiança e boa reputação.

Outra característica importante é que a maioria dos projetos bem-sucedidos foi iniciada por indivíduos, que tinham necessidades específicas, que não eram atendidas pelos produtos proprietários disponíveis. Para Bitzer et al. (2007), os mentores costumam iniciar projetos, por não estarem satisfeitos com as soluções existentes ou simplesmente porque o software necessário não existe. Um exmplo disso é Linus Torvalds, que queria uma versão do Unix para rodar em um PC e liderou o desenvolvimento do Linux.

\subsection{Motivações econômicas}

O envolvimento em projetos de Software Livre representa uma atividade de baixo custo, na qual o investimento financeiro para participar do processo é quase zero. Com a ampla cobertura da internet, os interessados podem contribuir do seu próprio computador enquanto estudam ou trabalham. [Osterloh et al. 2002].

Em termos de impactos na carreira profissional, o acúmulo de habilidades e experiências permite a evolução dos participantes que, por meio de suas contribuições, constroem uma reputação na comunidade e podem migrar para papéis centrais do 
modelo cebola [Jergensen et al. 2011]. A consequência natural da reputação alcançada é o ganho de prestígio e visibilidade, tornando a colaboração em projetos de Software Livre uma alternativa interessante para quem busca melhores posições no mercado de trabalho ou pensa em adotá-los como modelo de negócio [Lerner e Tirole 2002].

\subsection{Motivações sociopsicológicas}

Para Krogh et al. (2008), as motivações sociopsicológicas são semelhantes às motivações intrínsecas definidas por Gagné e Deci (2005), em que o interesse por uma atividade está associado à satisfação espontânea em realizá-la de forma voluntária.

Para Linus Torvalds, a maior motivação dos desenvolvedores é a diversão em programar [Ghosh 1998]. Raymond (1999) reforça que a diversão no desenvolvimento de soluções de código aberto pode nos ensinar um modo de trabalho criativo e economicamente mais eficiente. Mas, diversão em programar não é suficiente para compreender os motivos dos desenvolvedores de Software Livre, já que os programadores também poderiam se recusar a publicar o código-fonte [Bitzer et al. 2007]. Nesse sentido, outros motivos são apontados na literatura para justificar a liberação de um código sob licença livre.

Segundo Ljungberg (2000), as comunidades de código aberto são frequentemente analisadas como uma forma de 'cultura da dádiva'. Com base nessa cultura, todo presente ofertado está relacionado a uma obrigação de retribuição em algum momento futuro, mesmo que não seja uma exigência declarada. Na cultura da dádiva, a posição social não é determinada pelo que se detém ou controla, mas por aquilo que é doado. Sob essa perspectiva, presentear é uma forma de conquistar poder e controle. Os participantes dos projetos de Software Livre presenteiam toda a comunidade com informação ou conhecimento que, diferentes de bens tangíveis, se mantém com o desenvolvedor mesmo após a doação. Trata-se de um recurso infinito.

A cultura da dádiva oferece elementos para o 'altruísmo recíproco', no sentido de que os voluntários investem esforços para resolução de problemas na expectativa de estimular outros desenvolvedores a resolver problemas semelhantes e tornar as soluções públicas [Bitzer e Schrettl 2007].

O altruísmo puro, considerado como uma disposição pessoal oposta ao egoísmo, também está entre os fatores, que motivam a publicação de código-fonte [Hars e $\mathrm{Ou}$ 2001]. Richard Stallman (1985) ilustra esse espírito altruísta quando, fundamentado em princípios éticos, defende a liberdade que todos os usuários de computador têm de usar, estudar, copiar, modificar e redistribuir software.

Por fim, Hars e Ou (2001) destacam outra variante do altruísmo para explicar que a identificação com a comunidade também representa um estímulo para os voluntários. Denominado altruísmo de seleção de parentesco, esse tipo de motivação está vinculado à necessidade de ajudar pessoas que compartilham interesses ou características semelhantes. Por exemplo, Linus Torvalds declarou que tornar o código do Linux público foi uma decisão natural dentro da comunidade que ele sentia e da qual queria fazer parte [Ghosh 1998]. Esse tipo de altruísmo também está associado ao interesse, que muitas pessoas têm em colaborar para a melhoria da sociedade. 
V Congresso Brasileiro de Informática na Educação (CBIE 2016)

Anais do XXII Workshop de Informática na Escola (WIE 2016)

\section{Método}

\subsection{Contexto do estudo}

A pesquisa descrita neste artigo é um estudo de caso que, segundo Yin (2013), justificase em situações contemporâneas em que é necessária a investigação profunda de uma unidade de análise. Com o estudo, buscou-se analisar a efetividade do Núcleo SPB para motivar estudantes a colaborarem em projetos do Portal SPB. Nessa disciplina, os estudantes são apresentados a um conjunto de recursos e conceitos de Software Público e, além disso, realizam atividades práticas de colaboração em comunidades do Portal SPB. A descrição detalhada das atividades do Núcleo SPB pode ser acessada no trabalho de Silva (2014).

\subsection{Objetivo}

Analisar a efetividade do Núcleo SPB para motivar estudantes a colaborarem em projetos do Portal SPB.

\subsection{Participantes}

Os dados analisados nesta pesquisa são da primeira turma do Núcleo SPB, ofertada em 2014 pela Univasf. A disciplina possuía 40 estudantes matriculados. Dentre esses, apenas um dos estudantes não concluiu as atividades propostas na disciplina, em razão de um afastamento para a participação no programa Ciência sem Fronteira.

Tabela 1. Dados do perfil dos estudantes participantes da pesquisa

\begin{tabular}{l|l|c|c}
\hline \multicolumn{1}{c|}{ Questão } & \multicolumn{1}{|c|}{ Alternativa } & $\mathbf{N}^{*}$ & $\mathbf{\%}$ \\
\hline \multirow{2}{*}{ Qual a sua idade? } & Entre 18 e 20 anos & 04 & 10,26 \\
\cline { 2 - 4 } & Entre 21 e 23 anos & 17 & 43,59 \\
\cline { 2 - 4 } & 23 ou mais & 18 & 46,15 \\
\hline \multirow{2}{*}{ Qual o seu sexo? } & Feminino & 05 & 12,82 \\
\cline { 2 - 4 } & Masculino & 34 & 87,18 \\
\hline \multirow{2}{*}{ Qual o seu curso? } & Administração & 02 & 5,13 \\
\cline { 2 - 4 } & Engenharia Civil & 02 & 5,13 \\
\cline { 2 - 5 } & Engenharia da Computação & 22 & 56,41 \\
\cline { 2 - 5 } & Engenharia de Produção & 01 & 2,56 \\
\cline { 2 - 5 } & Engenharia Elétrica & 11 & 28,21 \\
\cline { 2 - 5 } & Engenharia Mecânica & 01 & 2,56 \\
\hline \multirow{2}{*}{ Já participou de comunidades de Software Livre? } & Sim & 04 & 10,26 \\
\cline { 2 - 5 } & Não & 35 & 89,74 \\
\hline \multirow{2}{*}{$* N$} & & &
\end{tabular}

* $N=$ Número de estudantes para cada alternativa.

A turma contou com estudantes de diversos cursos e que, em sua grande maioria $(89,74 \%)$, nunca tinha participado de comunidades de Software Livre (Tabela 1).

\subsection{Procedimentos}

Durante a pesquisa, os estudantes foram submetidos à aplicação de dois questionários de natureza quantitativa. O primeiro questionário foi aplicado antes da realização da disciplina com o objetivo de identificar o nível de motivação prévia dos estudantes para colaborarem no desenvolvimento do Software Público Brasileiro. O segundo teve a finalidade semelhante, mas, como foi submetido no final da disciplina, identificou a evolução de motivação dos estudantes após cursarem o Núcleo SPB. A comparação entre os resultados dos questionários aplicados em momentos distintos permitiu verificar, quantitativamente, a efetividade da disciplina em motivar os estudantes para colaborarem com o desenvolvimento do Software Público Brasileiro. 
V Congresso Brasileiro de Informática na Educação (CBIE 2016)

Anais do XXII Workshop de Informática na Escola (WIE 2016)

Os questionários foram estruturados com quatro questões sobre o perfil dos discentes (Tabela 1) e dezoito declarações associadas a escalas de Likert, para identificar a evolução da motivação dos estudantes em colaborarem com o Software Público Brasileiro. As declarações foram organizadas em blocos de três grandes áreas motivacionais definidas por Feller e Fitzgerald (2000): (i) Motivações sociopsicológicas: representadas pelas declarações de 05 até 10; (ii) Motivações tecnológicas: representadas pelas declarações de 11 até 16; (iii) Motivações econômicas: representadas pelas declarações de 17 até 21 .

As declarações utilizadas nos dois questionários são semelhantes, apenas com a diferença do tempo verbal das frases. As declarações do primeiro questionário estão listadas na Tabela 2.

Tabela 2. Declarações realizadas no inicio da disciplina.

\begin{tabular}{l|l}
\hline Código & \multicolumn{1}{c}{ Declaração } \\
\hline D05 & $\begin{array}{l}\text { Com o trabalho em um projeto de Software Público Brasileiro - SPB, espero ter maior consciência do potencial } \\
\text { do meu curso para o benefício da sociedade. }\end{array}$ \\
\hline D06 & Quero trabalhar em um projeto de SPB para beneficiar a sociedade. \\
\hline D07 & Participar de um projeto de SPB me inspira a usar as minhas habilidades para ajudar os outros. \\
\hline D08 & Tenho orgulho em colaborar com o SPB. \\
\hline D09 & Posso descrever as desvantagens e os benefícios do SPB. \\
\hline D10 & Espero gostar de trabalhar em um projeto de SPB porque posso contribuir para o desenvolvimento do país. \\
\hline D11 & Trabalhar em um projeto de SPB vai aumentar o meu interesse pela área de computação. \\
\hline D12 & $\begin{array}{l}\text { Espero gostar de trabalhar em um projeto de SPB porque me permite participar de uma comunidade } \\
\text { diversificada de desenvolvedores. }\end{array}$ \\
\hline D13 & Trabalhar em um projeto de SPB vai aumentar a minha autoconfiança na área de computação. \\
\hline D14 & Estou certo de que poderia participar do desenvolvimento de um projeto real de software. \\
\hline D15 & $\begin{array}{l}\text { Tenho certeza de que posso participar ativamente em uma comunidade do Portal SPB para desenvolver um } \\
\text { projeto de software. }\end{array}$ \\
\hline D16 & Espero ganhar alguma confiança em colaborar com profissionais de uma variedade de locais e culturas. \\
\hline D17 & Posso descrever as desvantagens e os benefícios do Software Público como modelo de negócio. \\
\hline D18 & $\begin{array}{l}\text { Participar de um projeto do Portal do SPB me ajudará a desenvolver uma postura profissional em um ambiente } \\
\text { de desenvolvimento de software. }\end{array}$ \\
\hline D19 & O SPB é muito relevante para os meus planos de carreira profissional. \\
\hline D20 & Posso aumentar minha reputação profissional ao colaborar com SPB. \\
\hline D21 & Tenho experiência em relação ao Software SPB. \\
\hline D22 & No geral, espero estar muito satisfeito com o meu aprendizado sobre SPB. \\
\hline &
\end{tabular}

Com base nos dados coletados por meio dos questionários, foram analisadas as seguintes hipóteses:

$\mathbf{H}_{1}$ : O Núcleo SPB causou impacto na motivação dos estudantes para colaborarem em projetos do Portal SPB.

$\mathbf{H}_{2}$ : O Núcleo SPB modificou a motivação inicial dos estudantes em relação ao SPB.

\section{Resultados}

Para avaliar os impactos do Núcleo SPB sobre a motivação dos estudantes em participar de comunidades do Portal SPB, nesta pesquisa, utilizamos como fonte de evidência os dados coletados por meio da aplicação de dois questionários, conforme indicado na Seção 3.4.

Os participantes da pesquisa utilizaram níveis de uma escala de Likert para avaliar declarações contidas nos questionários que, para fins de análise quantitativa, foram transformados de dados categóricos para dados numéricos ${ }^{1}$.

\footnotetext{
${ }^{1}$ 1: Discordo Completamente | 2: Discordo |3: Indiferente / Não Sei | 4: Concordo | 5: Concordo Completamente.
} 
V Congresso Brasileiro de Informática na Educação (CBIE 2016)

Anais do XXII Workshop de Informática na Escola (WIE 2016)

\subsection{Efetividade do Núcleo SPB}

O gráfico da Figura 1 apresenta indícios descritivos das respostas dos estudantes no questionário aplicado ao final da disciplina. A linha contínua representa a média das respostas fornecidas, enquanto a linha tracejada representa o nível neutro da escala de Likert (indiferente $=3$ ). Esse gráfico fornece uma retrospectiva sobre a experiência dos estudantes após participarem do Núcleo SPB e demonstra que as médias das respostas de todas as declarações (Tabela 2) estão acima do nível neutro.

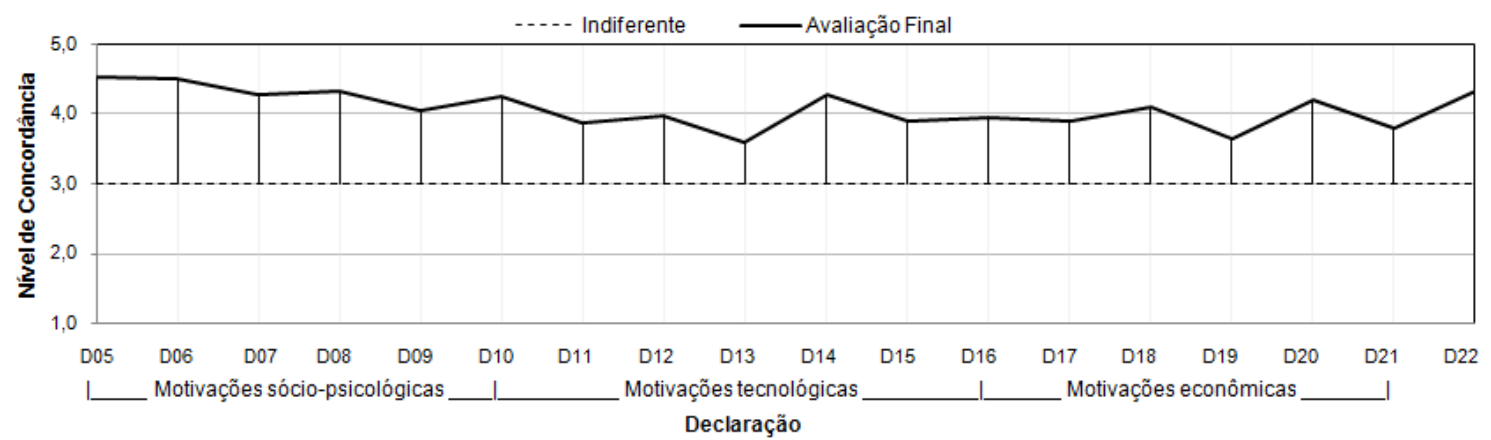

Figura 1. Média das respostas no questionário aplicado ao final do Núcleo SPB.

O item D22, com média 4,31 (acima do nível 'concordo'), evidencia que os estudantes ficaram muito satisfeitos com o aprendizado sobre Software Público Brasileiro. Entre os demais itens, as maiores médias de resposta estão relacionadas, principalmente, com os fatores sociopsicológicos. Os itens D05, D06, D07, D08, D09 e D10 revelam que o Núcleo SPB estimulou o altruísmo dos estudantes e forneceu-lhes informações suficientes para compreenderem, ainda mais, o potencial dos seus cursos e do SPB em beneficiar a sociedade.

A observação no gráfico da Figura 1 também demonstra que o Núcleo SPB parece ter estimulado positivamente os estudantes a reconhecerem motivações tecnológicas e econômicas no SPB, identificando oportunidades profissionais e oferecendo uma experiência em um projeto de software real e relevante para o país.

Embora as evidências dos impactos do Núcleo SPB sobre a motivação dos estudantes sejam perceptíveis graficamente, foram realizados testes estatísticos para verificação da seguinte hipótese:

$H_{1}:$ O Núcleo SPB causou impacto na motivação dos estudantes para colaborarem em projetos do Portal SPB.

Para verificar se as médias dos itens apresentavam diferenças significativas em relação ao nível neutro (hipótese $\mathbf{H}_{\mathbf{1}}$ ), foi utilizado o software estatístico $\mathrm{R}^{2}$ e o teste de Wilcoxon, com significância de 5\%. Esse teste é uma técnica não paramétrica, que tem como base de cálculo os postos (ranks) das diferenças intrapares, sendo recomendado para análise de dados ordinais emparelhados.

A Tabela 3 mostra que os resultados do teste de Wilcoxon, em todos os itens da pesquisa, a hipótese $\mathbf{H}_{\mathbf{1}}$ foi confirmada ( $p$-valor $<0,05$ em todas as declarações), apresentando fortes evidências de que o Núcleo SPB causou impacto na motivação dos estudantes para colaborarem em projetos do Portal SPB.

\footnotetext{
${ }^{2}$ http://www.r-project.org/
} 
V Congresso Brasileiro de Informática na Educação (CBIE 2016)

Anais do XXII Workshop de Informática na Escola (WIE 2016)

Tabela 3 - Teste de Wilcoxon para avaliar impacto do Núcleo SPB.

\begin{tabular}{c|c|c|c|c|c}
\hline Declaração & Média & $\boldsymbol{p}$-valor & Declaração & Média & $\boldsymbol{p}$-valor \\
\hline D08 & 4,51 & $4,0670 E-08$ & D17 & 4,26 & $1,0810 E-07$ \\
\hline D09 & 4,49 & $2,9696 E-08$ & D18 & 3,90 & $4,4252 E-06$ \\
\hline D10 & 4,26 & $9,2339 E-08$ & D19 & 3,95 & $5,9720 E-07$ \\
\hline D11 & 4,31 & $1,0125 E-07$ & D20 & 3,90 & $9,7473 E-06$ \\
\hline D12 & 4,05 & $5,7217 E-08$ & D21 & 4,08 & $2,4743 E-07$ \\
\hline D13 & 4,23 & $8,5709 E-08$ & D22 & 3,64 & $6,7874 E-04$ \\
\hline D14 & 3,87 & $2,6236 E-05$ & D23 & 4,18 & $3,7898 E-08$ \\
\hline D15 & 3,97 & $6,7381 E-07$ & D24 & 3,79 & $6,6826 E-06$ \\
\hline D16 & 3,59 & $4,8905 E-04$ & D25 & 4,31 & $1,8064 E-07$ \\
\hline
\end{tabular}

\subsection{Mudança na motivação inicial dos estudantes em relação ao SPB}

No gráfico da Figura 2, estão representadas as médias das repostas obtidas nos questionários aplicados no início e ao fim do Núcleo SPB. O gráfico apresenta evidências de algumas diferenças entre as expectativas iniciais e a avaliação final dos estudantes.

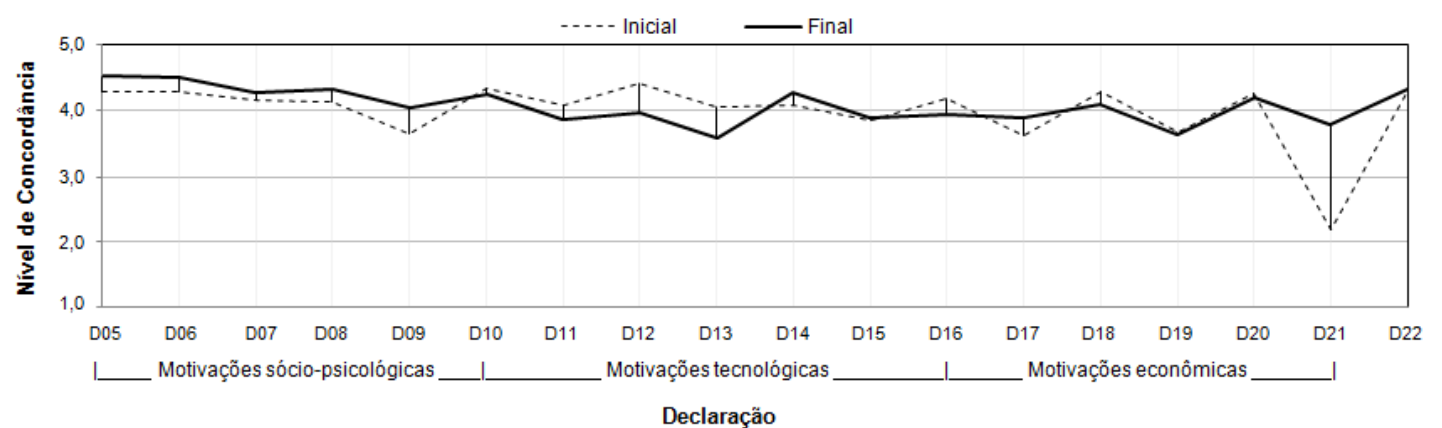

Figura 2. Resultado dos questionários inicial e final do Núcleo SPB.

Com o objetivo de verificar precisamente as diferenças significativas na motivação dos estudantes em relação ao SPB, foi realizado teste de Wilcoxon, comparando os resultados dos dois questionários para avaliar, em cada declaração, a seguinte hipótese:

$\mathrm{H}_{2}:$ O Núcleo $S P B$ modificou a motivação inicial dos estudantes em relação ao $S P B$.

Os resultados do teste de Wilcoxon (Tabela 4), a um nível de significância de $5 \%$, revelaram diferenças significativas (hipótese $\left.\mathrm{H}_{2}\right)$ em cinco itens $(p$-valor $<0,05)$ (Tabela 5).

Tabela 4 - Teste para avaliar a mudança de percepção em relação ao SPB.

\begin{tabular}{c|c|c|c|c|c|c|c}
\hline Declaração & Média inicial & Média final & $\boldsymbol{p}$-valor & Declaração & Média inicial & Média final & $\boldsymbol{p}$-valor \\
\hline D05 & 4,28 & 4,51 & $4,9535 E-02$ & D17 & 4,08 & 4,26 & $1,8236 E-01$ \\
\hline D06 & 4,28 & 4,49 & $8,8082 E-02$ & D15 & 3,85 & 3,90 & $7,8354 E-01$ \\
\hline D07 & 4,15 & 4,26 & $4,0526 E-01$ & D16 & 4,18 & 3,95 & $1,4515 E-01$ \\
\hline D08 & 4,13 & 4,31 & $1,2663 E-01$ & D17 & 3,62 & 3,90 & $1,2039 E-01$ \\
\hline D09 & 3,64 & 4,05 & $7,0667 E-03$ & D18 & 4,28 & 4,08 & $1,1666 E-01$ \\
\hline D10 & 4,33 & 4,23 & $4,3277 E-01$ & D20 & 3,67 & 3,64 & $8,8413 E-01$ \\
\hline D11 & 4,08 & 3,87 & $1,3847 E-01$ & D21 & 4,26 & 4,18 & $5,7960 E-01$ \\
\hline D12 & 4,41 & 3,97 & $2,3090 E-03$ & D22 & 2,21 & 3,79 & $8,2960 E-07$ \\
\hline D13 & 4,05 & 3,59 & $1,4558 E-02$ & D23 & 4,31 & 4,31 & $8,8862 E-01$ \\
\hline
\end{tabular}

Combinando as informações do gráfico da Figura 2 e os resultados do teste de Wilcoxon (Tabela 4), nota-se que as evidências do ganho em motivações sociopsicológicas foram reforçadas. A análise dos itens D05 e D09 demonstra que a expectativa dos estudantes foi superada em relação ao potencial de seus cursos e do SPB em beneficiar a sociedade. Também ficou evidente que as atividades realizadas no 
V Congresso Brasileiro de Informática na Educação (CBIE 2016)

Anais do XXII Workshop de Informática na Escola (WIE 2016)

Núcleo SPB contribuíram para os estudantes ganharem experiência em Software Público Brasileiro, fazendo com que a média das respostas do item D21 mudasse de 2,21 para 3,79 (Tabela 5).

Tabela 5 - Declarações em que os estudantes mudaram de percepção.

\begin{tabular}{l|l}
\hline D05 & $\begin{array}{l}\text { Com o trabalho em um projeto de SPB Brasileiro, tenho maior consciência do potencial do meu curso } \\
\text { para o benefício da sociedade. }\end{array}$ \\
\hline D09 & Posso descrever as desvantagens e os benefícios do SPB para a sociedade. \\
\hline D12 & $\begin{array}{l}\text { Gostei de trabalhar em um projeto de SPB porque me permitiu participar de uma comunidade } \\
\text { diversificada de desenvolvedores. }\end{array}$ \\
\hline D13 & Trabalhar em um projeto de SPB aumentou a minha autoconfiança na área de computação. \\
\hline D21 & Tenho experiência em relação ao SPB. \\
\hline
\end{tabular}

Apenas nos itens D12 e D13, houve pequenas diferenças negativas entre as médias inicial e final. As declarações tratam do trabalho em uma comunidade diversificada de desenvolvedores e aumento da autoconfiança na área de computação. $\mathrm{Na}$ próxima seção, por meio dos resultados da entrevista online com grupo focal, discutimos as causas dessas reduções nas médias. Em uma entrevista conduzida com um grupo focal, os estudantes revelaram que a redução na avaliação desses itens foi causada, principalmente, pela baixa interação percebida nas comunidades do Portal SPB e pelas dificuldades encontradas na instalação dos softwares.

\section{Considerações Finais}

Este trabalho, por meio de um estudo de caso, analisou a efetividade da componente curricular Núcleo SPB para motivar a participação de estudantes em comunidades virtuais do Portal SPB.

Ao final do semestre letivo, mais de $90 \%$ dos participantes da pesquisa se declararam muito satisfeitos com o conhecimento adquirido na componente curricular. As análises descritivas das respostas dos questionários e testes inferenciais, especificamente, os testes não paramétricos de Wilcoxon, permitiram verificar uma evolução significativa na motivação dos discentes em relação a fatores sociopsicológicos, tecnológicos e econômicos associados ao Software Público Brasileiro.

Apesar de ter alcançado o objetivo estabelecido nesta pesquisa, vários outros trabalhos podem ser realizados para aprofundar os estudos sobre a adoção de Software Público em atividades acadêmicas, entre eles podemos citar: (i) Realizar pesquisas sobre os impactos do Software Público Brasileiro na carreira profissional dos estudantes egressos do Núcleo SPB; (ii) Investigar se a participação em comunidades do Portal SPB estimula o interesse dos estudantes por seus cursos de graduação.

\section{Agradecimentos}

Esta pesquisa é uma ação do Programa de Formação de Agentes para Sustentabilidade do Software Público Brasileiro, programa de extensão coordenado pela UFPE em parceria com a Univasf, realizado com o apoio do PROEXT - MEC/SESu.

\section{Referências}

Bitzer, J., Schrettl, W., Schröder, P. (2007). "Intrinsic motivation in open source software development". Journal of Comparative Economics, v. 35, n. 1, p. 160-169. 
V Congresso Brasileiro de Informática na Educação (CBIE 2016)

Anais do XXII Workshop de Informática na Escola (WIE 2016)

Bonaccorsi, A., Rossi, C. (2006). "Comparing motivations of individual programmers and firms to take part in the open source movement: From community to business". Knowledge, Technology \& Policy, v. 18, n. 4, p. 40-64.

Brasil. Ministério da Educação. (2015). "Edital Proext/MEC 2016". Disponível em: < http://portal.mec.gov.br/component/content/article?id=12243:editais $>$. Acesso em: 17/05/16.

Feller, J., Fitzgerald, B. (2000). "A framework analysis of the open source software development paradigm". In: Proceedings of the twenty first international conference on Information systems. Association for Information Systems. p. 58-69.

Gagné, M., Deci, E. (2005). "Self-determination theory and work motivation". Journal of Organizational behavior, v. 26, n. 4, p. 331-362.

Ghosh, R. (1998). "Interviews with Linus Torvalds: What motivates software developers". First Monday, v. 3, n. 2.

González, J. et al. (2007). "Introducción al software libre". Universitat Oberta de Catalunya.

Hars, A., Ou, S. (2001) "Working for free? Motivations of participating in open source projects". In: System Sciences, 2001. Proceedings of the 34th Annual Hawaii International Conference on. IEEE, 2001. p. 9 pp.

Jergensen, C., Sarma, A., Wagstrom, P. (2011). "The onion patch: migration in open source ecosystems". In: Proceedings of the 19th ACM SIGSOFT symposium and the 13th European conference on Foundations of software engineering. ACM, p. 70-80.

Ljungberg, J. (2000). "Open source movements as a model for organizing". European Journal of Information Systems, v. 9, n. 4, p. 208-216.

Lerner, J., Tirole, J. (2002). "The open source movement: Key research questions". European Economic Review, v. 45, n. 4, p. 819-826.

Osterloh, M., Rota, S., Kuster, B. (2002). "Trust and Commerce in Open Source-a Contradiction?". Trust in the Network Economy, p. 129-141.

Pinto, G., Kamei, F. (2014). "The Census of the Brazilian Open-Source Community". In: Open Source Software: Mobile Open Source Technologies. Springer Berlin.

Raymond, E. (1999). "The cathedral and the bazaar". Knowledge, Technology \& Policy, v. 12 , n. 3, p. 23-49.

Scacchi, W. et al. (2006). "Understanding free/open source software development processes". Software Process: Improvement and Practice, v. 11, n. 2, p. 95-105.

Silva, J. (2014). "Análise da Efetividade de Componente Curricular Para Motivar Colaboradores em Projetos de Software Público Brasileiro". Disponível em: http://repositorio.ufpe.br:8080/xmlui/handle/123456789/11532. Acesso em: 17/05/16.

Sommerville, I. (2011). Engenharia de Software. 9. ed. São Paulo: Pearson Prentice Hall. $544 \mathrm{f}$.

Stallman, R. The GNU Manifesto. 1985. Disponível em: http://www.gnu.org/gnu/manifesto.html. Acesso em 20/05/2016.

Terceiro, A. et al.(2015). "Software Público Brasileiro: de portal para plataforma integrada de colaboração". Revista da Sociedade Brasileira de Computação.

Krogh, G. et al. (2008). "Open source software: What we know (and do not know) about motivations to contribute". In: The DRUID Conference: 17 June 2008; Copenhagen, Denmark.

Yin, R. K. (2013). “Case study research: Design and methods”. Sage publications. 